\title{
Kalman Filter and Wavelets Transform Based Three-Phase Power Quality Disturbances Detection, Classification and Diagnosis Tool Implementation - Hardware and Software
}

\author{
Alexandre A. Carniato, Ruben B. Godoy, João Onofre P. Pinto \\ Department of Electrical Engineering \\ DEL., Federal University of Mato Grosso do Sul \\ BATLAB - Laboratory of Artificial Intelligence, Digital Systems and Power Electronics \\ Campus of Campo Grande - MS, \\ Phone/Fax number: +55 67 3345-3673, e-mail: alexandre@batlab.ufms.br
}

\begin{abstract}
The aim of this work is the development of a three-phase power quality disturbances detection, classification and diagnosis tool. The tool senses the electrical grid, and as a disturbance is detected, the voltage signals are acquired and analyzed. The result of the analysis is the classification of the disturbance and the diagnostic of its probable causes. The detection is done using Kalman Filter, while the classification and diagnosis are done using wavelets and fast Fourier transforms. The implementation involves hardware and software. The hardware is composed by voltage sensors, signal conditioning circuit, DSP320C6713 DSP board and an acquisition board. The software is responsible for the classification and diagnosis. Three cases of typical disturbances that affect electrical systems are presented. The results are consistent showing the feasibility of the proposed tool.
\end{abstract}

\section{Key words}

Three phase power quality disturbances, signal processing, classification, diagnosis

\section{Introduction}

R\&D addressing power quality issues have increased substantially during the last years. This fact, it can be explained based on: the increase of embedded generation and new renewable sources, the increasing sensitivity of new equipments to disturbances, and the increase of nonlinear loads. The detection and classification of problems related to power quality (PQ) have become a challenge for the researches. Many methods aiming to obtain solutions to detect and to classify disturbances have been proposed lately. The existent methods are based on visual waveform analysis and they can not be used in real time applications [1]. The recent advances of the signal processing have made possible to develop methods that are more reliable, as proposed by [1]-[4].

This work presents the development of a tool that, detects, classifies and diagnoses real voltage disturbances observed in electrical grid. For the terminology consistency sake, it is important to define the terms detection, classification and diagnoses used in this paper. The disturbance detection algorithm identifies the disturbance and determines its duration, the classification algorithm, in its turn, classifies the disturbance type, as harmonics, sags, swell etc. Finally, the diagnosis algorithm identifies the probable disturbance source.

The tool that will be presented in this paper, called PQMON, is composed by hardware and software. The hardware has 4 components: sensors, signal conditioning circuit, DSP board, data acquisition board. The software, which runs in a PC, classifies the disturbance and makes the diagnostics of what is causing it.

\section{Categories of Three-Phase Power Quality Disturbances}

The power quality disturbances classification and diagnosis methodologies for three-phase systems are more complex than the methodologies for single-phase systems presented in [5]. So, the classification and diagnoses algorithms for three-phase systems were developed considering the only most relevant disturbances, i. e., disturbances that have more occurrences in electrical systems. These disturbances are: Harmonics, Sags, Swells and Interruptions [6]. Figure 1 shows the disturbances aforementioned. As it can be seen in Figure 1a it shows the presence of harmonics, while in Figure 1b, single-phase sag event is shown. In Figure 1c, a single-phase swell is presented, and finally in Figure 1d, a typical case of interruption is given. Detailed descriptions of these disturbances are provided in [5],[8].

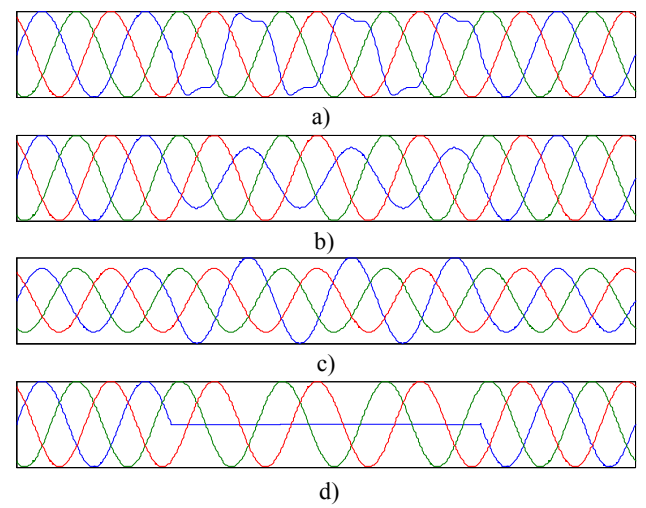

Figure 1. Typical three-phase power quality disturbances. 


\section{System Overview}

This section will present in details the proposed tool. Figure 2 shows the block diagram of such system. The system is composed by hardware and software.

\subsection{Hardware}

The hardware part is composed by three blocks, as it could be seen in figure 2. In the first one (signal conditioning), there are three voltage sensors, low passfilters and buffers. The sensors chosen were LV-25P, because they are based on Hall Effect. This characteristic is very important due the linearity of response in a large range of frequency. To do the sampling of a constant frequency signal, it could be used a simple transformer, nevertheless in this application, they will not have a reliable response due the possibility of existence of highfrequency disturbances, like, oscillatory/impulsive transients and harmonics.

The low-pass filter was configured with a cut-off frequency of $7680 \mathrm{~Hz}$. This value was chosen due the sampling rate used in the software to do the classification and diagnosis.

The buffer is an extremely useful circuit, since it helps to solve impedances issues. As it known, the impedance difference guarantee isolation between the signal from electrical grid and the signal read by analog-digital converter of DSP.

The second block is called DSP. The Kalman filter runs in a DSP from Texas Instruments. The Kalman filter was used as a synchronism algorithm, and more details will be presented in the next section.

The signals from electrical grid and the signals from Kalman filter are captured using an acquisition board, showed in third block dashed in figure 2. In the sequence, these signals are used to detect a probable occurrence. The error between these signals is calculated, and if this value is greater than a threshold, these data are saved to be processed in the software of classification and diagnosis.

\subsection{Software}

The software runs in a PC based on Matlab/Simulink code. This software is able to detect and classify fourteen types of electrical three-phase disturbances. The wavelet and FFT are used to do this classification. The diagnosis of three-phase disturbances are made based on the behavior of three-phase voltages, and in the future, the neutral current could be add as a new variable to achieve more reliable diagnosis.

This topology, using a PC for processing signals, was used for fast validation of the methodology, however, the ultimate goal is an equipment for stand-alone operation. Thus, the PC and data acquisition board will be removed from the system and a display will be added, as showed in the blue dashed box in Figure 2. In that case, the whole software will run the DSP board, and the results will be directly presented in the display.

\section{Mathematical Tools}

The mathematical tools used in this work to detect and classify disturbances were Wavelet transform, FFT, and some statistic tools. The algorithms to do detection and classification basically use a reference signal for comparisons propose. This reference signal it has to be synchronized with the electric grid voltage. So, the algorithm compares the sensed three-phase signals to the synchronized reference signal, and the errors are used to detect the existence of disturbances. The synchronization algorithm is based on Kalman Filter theory.

The Kalman filter algorithm is based on linear systems, so, the first step is to develop a model that describes the dynamic behavior of an electrical grid. A linear system can be described by two expressions showed below.

$$
\begin{gathered}
x_{k+1}=A x_{k}+B u_{k}+w_{k} \\
y_{k}=C x_{k}+z_{k}
\end{gathered}
$$

In the expressions above, the terms $\mathrm{A}, \mathrm{B}$, and $\mathrm{C}$ are matrices, " $\mathrm{x}$ " is the state of system, index " $\mathrm{k}$ " is the step time and "wk" and "yk" are noises. An electrical signal can be modeled based in two state variables. Thus, one possible model for an electrical grid is showed in (3) and (4).

$$
\begin{gathered}
{\left[\begin{array}{l}
x_{1} \\
x_{2}
\end{array}\right]_{k+1}=\left[\begin{array}{cc}
\cos \left(w_{k} T_{s}\right) & \operatorname{sen}\left(w_{k} T_{s}\right) \\
-\operatorname{sen}\left(w_{k} T_{s}\right) & \cos \left(w_{k} T_{s}\right)
\end{array}\right]\left[\begin{array}{l}
x_{1} \\
x_{2}
\end{array}\right]_{k}+\left[\begin{array}{l}
w_{1} \\
w_{2}
\end{array}\right]_{k}} \\
y_{k}=\left[\begin{array}{ll}
1 & 0
\end{array}\right]\left[\begin{array}{l}
1 \\
x 2
\end{array}\right]_{k}+v_{k}
\end{gathered}
$$

After this, the theory of Kalman Filter can be applied. The Kalman Filter is an algorithm of digital synchronism, which estimates the state based on the output and noisy measurements. The random variables "wk" and "zk" represent respectively the process and measurement noise. These noises are assumed to be independent (of each other), white, and with normal probability distributions. Their covariance matrices are defined as follow in expressions (5) and (6):

$$
\begin{aligned}
& \operatorname{cov}\left(w_{k}\right)=E\left(w_{k} \cdot w_{k}{ }^{T}\right)=Q \\
& \operatorname{cov}\left(z_{k}\right)=E\left(z_{k} \cdot z_{k}{ }^{T}\right)=R
\end{aligned}
$$

The matrices $\mathrm{R}$ and $\mathrm{Q}$ are the process noise covariance matrix and measurement noise covariance matrix, 


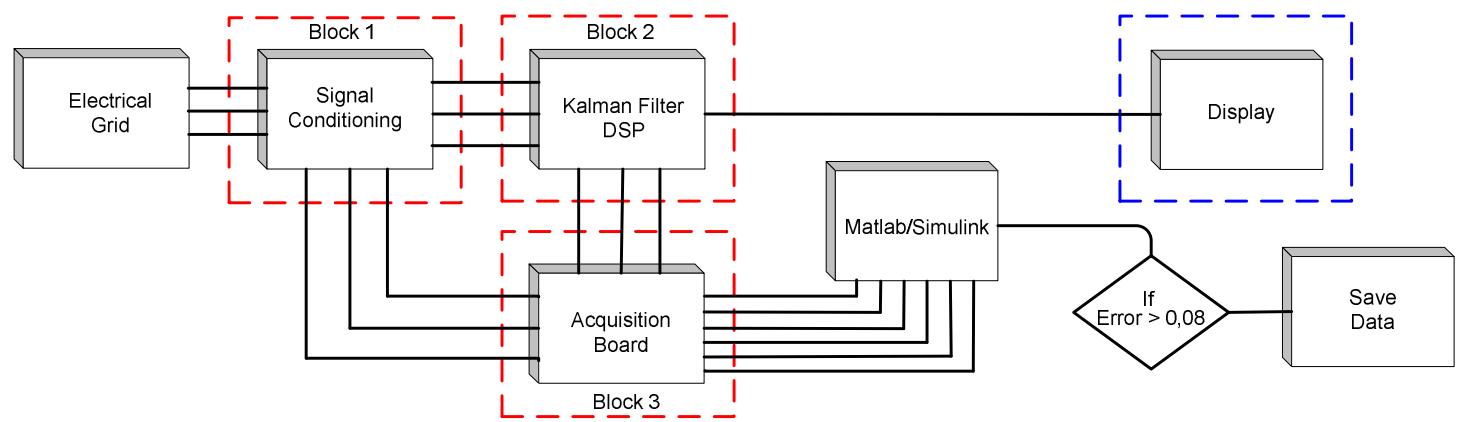

Figure 2. Block diagram of proposed system.

respectively. Finally, the equations of Kalman Filter can be introduced. There are many alternative however equivalent ways to express the equations. Among the amount of possibilities, one was chosen as follows in (7), (8) and (9).

$$
\begin{gathered}
K_{k}=A P_{k} C^{T}\left(C P_{k} C^{T}+R\right)^{-1} \\
\hat{x}_{k+1}=\left(A \hat{x}_{k}+B u_{k}\right)+K_{k}\left(y_{k+1}-C \hat{x}_{k}\right) \\
P_{k+1}=A P_{k} A^{T}+Q-A P_{k} C^{T} R^{-1} C P_{k} A^{T}
\end{gathered}
$$

Resuming, it consists of three-equations involving matrix manipulation. The matrix $\mathrm{K}$ is called Kalman Gain and $\mathrm{P}$ is called estimation error covariance.

The expression (8) is the state estimate equation. The first term use the influence of the state in a past time to derive the estimate state variable at time $\mathrm{k}+1$. The Kalman gain assesses the importance of measured output in the estimation of the state at time $\mathrm{k}+1$. If the Kalman gain is small, the output will not affect so much the estimation. This second term is called correction term.

Analyzing the expression (5), it could be seen if the measurement noise is small, so, the noise measurement covariance matrix will be small, and the Kalman gain will be large. In this case, the influence of output measurement in computation of state variables has a lot of credibility. In the other hand, if the noise measurement is large, the noise measurement matrix will be large too, so, the Kalman gain will be small, and the influence of measurement at the state estimation will be small.

The Kalman filter was implemented in a DSP from Texas Instruments. The code was created using the Matlab/Simulink environment.

In the future it is aimed a stand-alone operation, that will bring in a board a DSP that will loaded with a code that will classify and detect the fourteen types of disturbances detected in this prototype.

\section{Results and Analysis}

In order to evaluate the proposed tool, some experiments were done. Figure 3 presents a case where a disturbance was detected and classified as a "Sag in all the three phases". The diagnostic algorithm found as probable sources of this disturbance: "Three phase fault" or "Motor Start up", as shown in Figure 4.

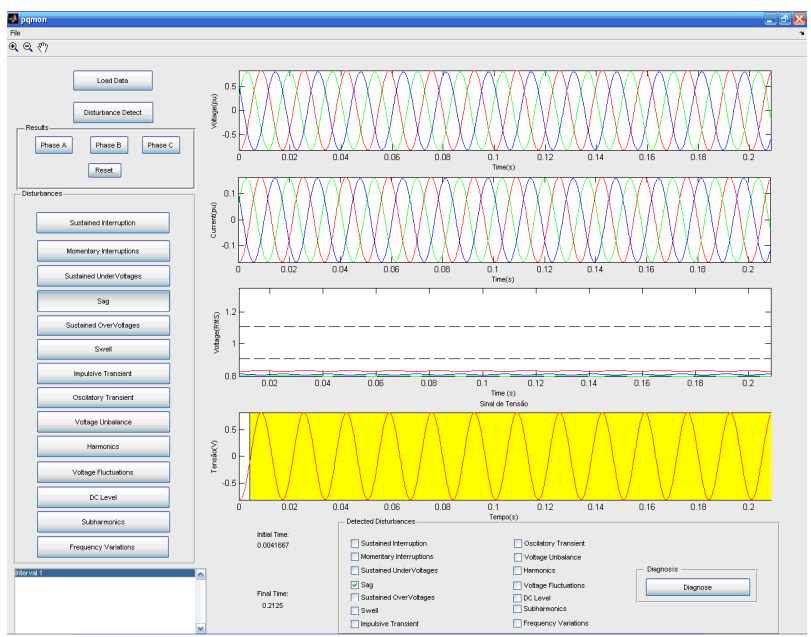

Figure 3. Three phase sag after PQMON analysis.

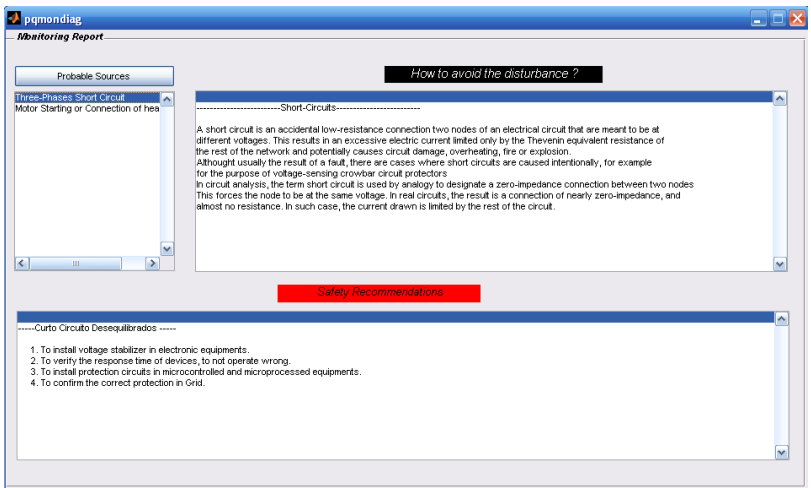

Figure 4. Disturbance probable sources

Figure 5 presents another experiment, and it displays the disturbance. Although from the first plot (sensed voltage) is not possible to positively identify the disturbance, from the third plot it can be observed a not standard behavior of the RMS value evolution, cycle by cycle, of phase A. The used signals processing algorithms suggest that there is a harmonic injection in phase A.

Among the probable sources the software identifies two possibilities: Non-Linear Loads and/or Energizing of transformers. These classes of sources are displayed in superior right side in Figure 6. Two remarks should be pointed out to support the software decision. The first one is related to the current behavior. It was not observed any current transient, which it is common in transformer 
energizing. Another remark is related to the number of occurrences in electrical systems, i. e., it is important to rank the sources in respect to the number of occurrences. From these two remarks, considering the software output, it can be deduced that the probable disturbance source is a Non-Linear Load. In this case, the load injects harmonic currents into the grid.

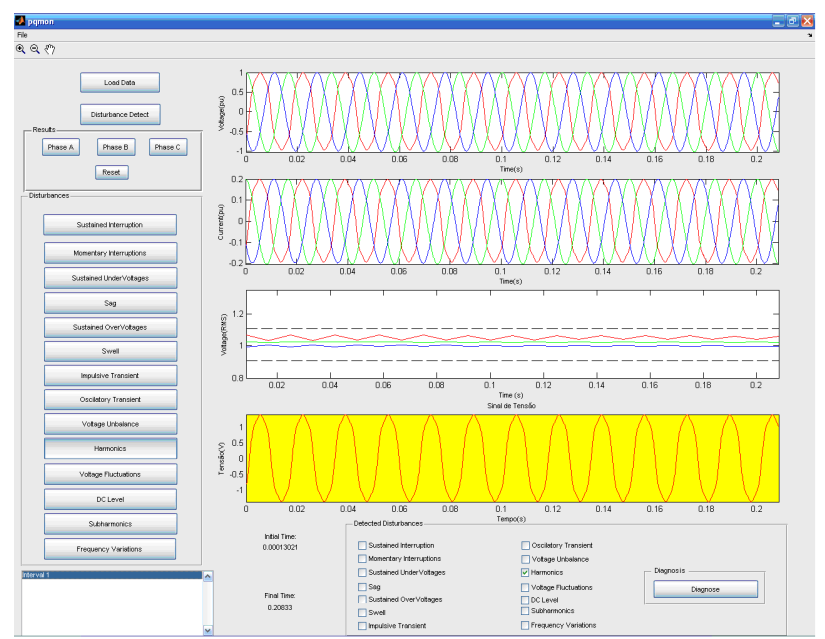

Figure 5. PQMON analysis results of a signal containing harmonics.

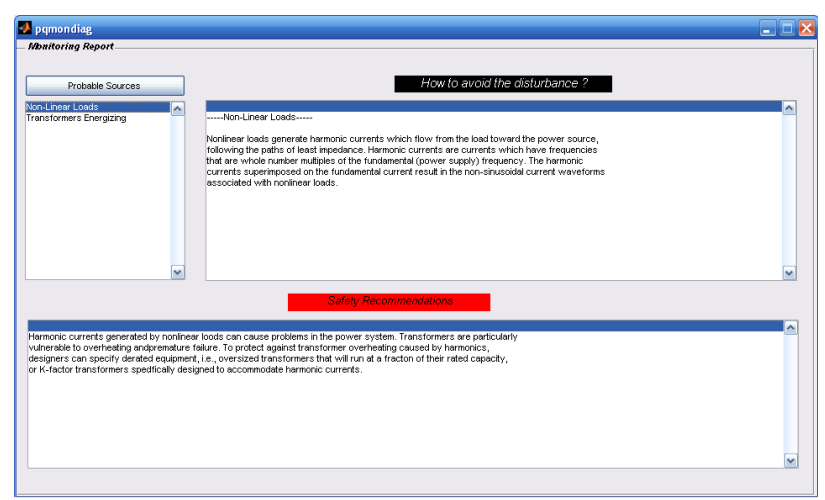

Figure 6. Probable causes of harmonic

In figure 7 is showed a typical case of interruption. The voltage magnitude in phase $\mathrm{A}$ is almost zero during a few cycles. This disturbance is known as Momentary Interruption. The most typical sources for this disturbance are: short-circuits, incorret operation of protection devices [8]. Figure 8 shows the software diagnosis for this disturbance and there are some recommendations to avoid and mitigate it [8].

\section{Conclusions}

In this work, it was discussed the implementation of a system that detects, classifies and makes the diagnosis of three-phase power quality disturbances. The detection strategy is performed based in a reference signal synchronized with the grid through a Kalman filter.

Signal processing and statistical techniques were used to classify the disturbances. The three-phase diagnosis presents much more variables compared to single-phase diagnosis. Thus, the algorithm formulation is more elaborated. This equipment was developed to detect, classify and diagnose fourteen types of three-phase disturbances. The insertion of new variables to make the diagnose more reliable has been studied. The neutral current may be used in the future to determine more accurately a specific disturbance source. Another variable, line current, can also be used to improve software diagnosis. Finally, it is aimed a hardware implementation for stand-alone operation, which will be addressed in the future.

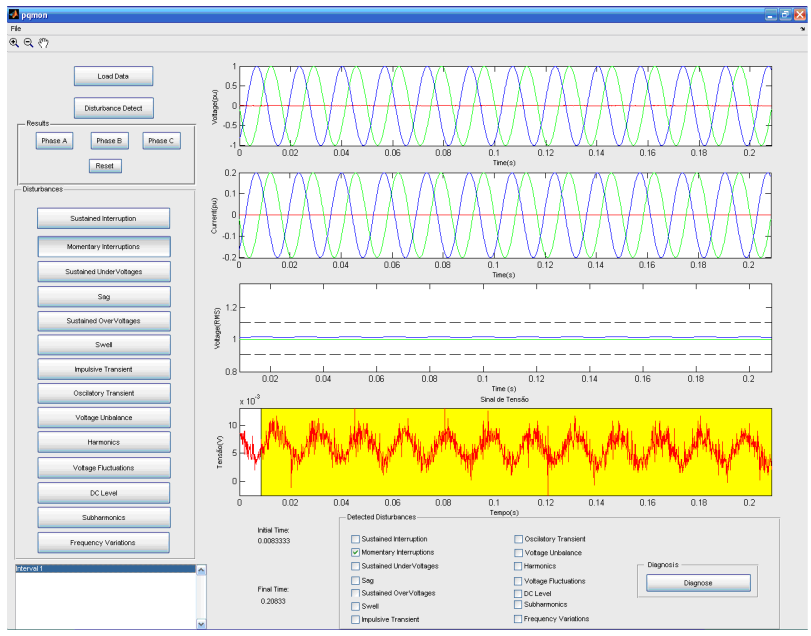

Figure 7. Signal with a single-phase interruption.

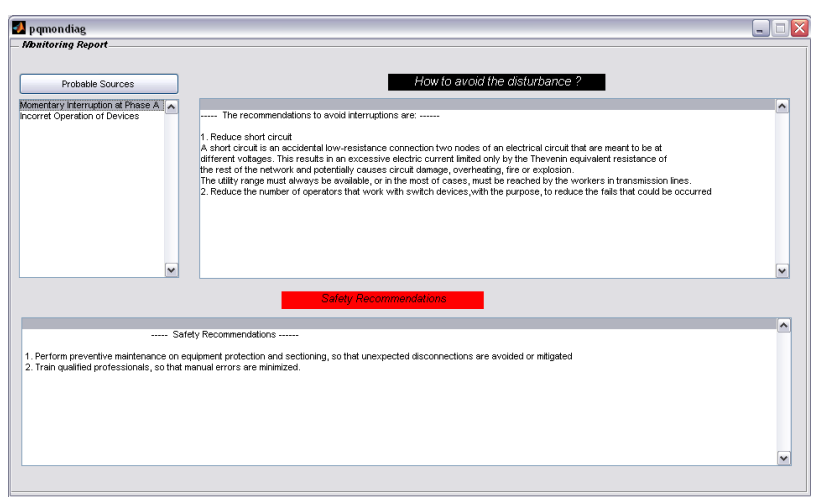

Figure 8. Probable cause of a single-phase interruption.

\section{References}

[1] S. Santoso, E. J. Powers, W. M. Grady, A. C. Parsons, "Power quality disturbance waveform recognition using wavelet-based neural classifier. I. Theoretical foundation." IEEE Transactions on Power Delivery, Vol. 15, pp. 222-228, Jan. 2000.

[2] A. M. Gaouda, M. M. A. Salama, M. R. Sultan, A. Y. Chikhani, "Power quality detection and classification using wavelet-multiresolution signal decomposition," IEEE Transactions On Power Delivery, Vol. 14, pp.14691476, Oct. 1999.

[3] I. Y. Chung, D. J. Won, J. M. Kim, et al. "Development of power quality diagnosis system for power quality improvement," IEEE Power Engineering Society General Meeting, vol.2, p. 1261, July 2003.

[4] J. S. Lee, C. H. Lee, J. O. Kim, S. W. Nam, "Classification of power quality disturbances using 
orthogonal polynomial approximation and bispectra," Electronics Letters, Vol. 33, pp. 1522-1524, Aug. 1997.

[5] Godoy, R.B.; Pinto, J.O.P.; Galotto, L., "Multiple signal processing techniques based power quality disturbance detection, classification, and diagnostic software," Electrical Power Quality and Utilisation, 2007. EPQU 2007. 9th International Conference on , vol., no., pp.1-6, 9-11 Oct. 2007.

[6] Dugan, R. C., Mcgranaghan, M. F. and Beaty, H. W. "Electrical power systems quality." New York: McGraw-Hill, xv, 265p. 1996.

[7] Kalman, R. E. “A New Approach to Linear Filtering and Prediction Problems," Transaction of the ASMEJournal of Basic Engineering, pp. 35-45 (March 1960).

[8] Bollen.M.H.J, Gu. I. Y. H., "Signal Processing Of Power Quality Disturbances", Wiley-Interscience, Piscataway (2006), pp. 41-161. 